\title{
The impact of flexibility on flow, turbulence and vertical mixing in coastal canopies
}

Maryam Abdolahpour1, 2, 3, *

Marco Ghisalberti1, 2

Kathryn McMahon3

Paul Lavery3

1School of Civil, Environmental and Mining Engineering, The University of Western Australia, Perth, WA 6009, Australia.

2Department of Infrastructure Engineering, School of Engineering, The University of Melbourne, Parkville, VIC 3010, Australia.

3School of Science, Edith Cowan University, Joondalup, WA 6027, Australia.

Corresponding author: M. Abdolahpour, School of Civil, Environmental and Mining Engineering, The University of Western Australia, Perth, WA 6009, Australia. (maryam.abdolahpour@uwa.edu.au)

\section{Abdolahpour et al.}

\section{The impact of flexibility on oscillatory canopy flow}

abstract Physical modeling of canopy-flow interactions has mostly employed rigid model vegetation, whereby the canopy geometry (i.e., its height and frontal area) is invariant and easily quantified. Here, we demonstrate that embedding realism in model vegetation, in the form of buoyancy and flexibility, can profoundly impact the structure of the flow and rates of vertical mixing in wave-dominated conditions. A laboratory investigation was undertaken with two types of model canopy: (1) rigid canopies consisting of wooden dowels, and (2) flexible,

This is the author manuscript accepted for publication and has undergone full peer review but has not been through the copyediting, typesetting, pagination and proofreading process, which may lead to differences between this version and the Version of Record. Please cite this article as doi: 10.1002/lno.11008

This article is protected by copyright. All rights reserved. 
buoyant model plants designed to mimic meadows of the seagrass Posidonia australis. To isolate the impact of flexibility, the maximum heights and frontal areas of the two types of canopy were matched. These canopies were subjected to oscillatory flows with a realistic range of wave heights and periods. Drag reduction caused by the reconfiguration of flexible canopies leads to a greatly diminished velocity attenuation in the canopy (on average 65\%). The reduced average height of flexible canopies shifts the canopy shear layer towards the bed, resulting in significantly enhanced levels of near-bed turbulence. Finally, a decreased vertical diffusivity (approximately 35\%) was observed in the flexible model canopies, relative to the rigid analogues. Thus, while the use of dynamically-scaled vegetation adds complexity to modeling efforts, it represents a step towards a more accurate quantitative understanding of flow and mixing in these environments.

\section{Introduction}

Coastal ecosystems are often characterized by large and complex benthic roughness formed by, e.g., seagrass meadows, coral communities and kelp forests (Duarte, 2002; Rosman et al., 2007; Barbier et al., 2011). These 'canopies' provide food (Connolly et al., 2005), nursery, habitat (Fonseca et al., 1992) and shelter (Scott et al., 2000) for a wide range of marine organisms. Canopies may also oxygenate the water (Larkum et al., 2006) and play an essential role in maintaining water clarity through the uptake of organic matter and capture of particulate material (Moore, 2004; Larkum et al., 2006). The drag exerted by the canopy also attenuates the wave energy and in-canopy velocity (Kobayashi et al., 1993; Manca et al., 2012). Thus, coastal canopies reduce the resuspension of particulate material (Hansen and Reidenbach, 2011) and enhance sedimentation (Gacia et al., 1999), carbon burial (Granata et al., 2001) and the retention of dissolved and particulate material within the canopy (Fonseca and Cahalan, 1992; Granata et al., 2001).

Coastal canopies are often flexible, an advantage which allows them to adapt their posture in response to the flow and reduce the drag (Rominger and Nepf, 2014), thus preventing uprooting during periods of strong currents and extreme waves (Vogel, 1984; Albayrak et al., 2012). In currentdominated conditions, this drag reduction due to flexibility results in a diminished velocity attenuation inside the canopy (Nepf, 2012a,b) and therefore a weaker shear layer at the top of the canopy (Ghisalberti and Nepf, 2006). Moreover, coherent canopy waving (known as 'monami', Ackerman and Okubo (1993)) has been observed to result in a 40\% reduction in canopy drag and diminished vertical mixing of momentum

This article is protected by copyright. All rights reserved. 
across the top of the canopy (Ghisalberti and Nepf, 2006, 2009). In oscillatory flows, the time-varying roughness that results from canopy oscillation may cause a substantial drag reduction relative to rigid upright canopies (Luhar and Nepf, 2016). Hydrodynamic drag forces in stiff canopies have been shown to be three times higher than that in canopies with flexible leaves (Bouma et al., 2005). As a result, canopy flexibility has been observed to greatly affect the extent of wave attenuation (Paul et al., 2012; Houser et al., 2014).

Canopy flexibility can also have important implications for chemical and biological processes. For example, the orientation of seagrass blades can influence light availability within the meadow, such that an increase in bending from 5 to 20 degrees greatly enhances canopy photosynthesis (Zimmerman, 2003). Flexibility has also been shown to positively influence nutrient uptake (Hurd, 2000; Huang et al., 2011) and efflux of dissolved oxygen from canopy elements (Mass et al., 2010). This is attributed to the reorientation, pitching and flapping of the blade in response to the flow, which can lead to a thinning or removal of the diffusive boundary layer around the blade (Denny and Roberson, 2002; Stevens et al., 2003).

There has been growing interest in the structure of oscillatory (Pujol et al., 2013a) and turbulent flows (Reidenbach et al., 2007; Pujol et al., 2013b) in submerged canopies, as well as in understanding vertical mass (Nishihara et al., 2011; Abdolahpour et al., 2017a) and momentum transport (Ghisalberti and Schlosser, 2013). The majority of physical and numerical modeling efforts have used rigid cylinders to simulate canopy elements, as this allows the canopy geometry to be invariant over time and therefore easily quantified. These rigid elements may reasonably represent stem-like aquatic vegetation and hard corals, but do not successfully mimic canopies where flexibility, buoyancy and reconfiguration are important (Koehl et al., 2008; Mass et al., 2010).

\section{Hydrodynamics of oscillatory canopy flows}

The drag exerted by the canopy results in a vertical gradient of orbital velocity across the canopy-water interface. That is, the root-mean-square (RMS) of the oscillatory velocity within the canopy, $U_{c}^{r m s}$ (where subscript ' $c$ ' refers to the in-canopy average), is attenuated from its value far 
above the canopy, $U_{\infty}^{r m s}$. This velocity shear, which is greater for denser canopies (Abdolahpour, 2017), can lead to the generation of KelvinHelmholtz-type vortices (KH-vortices) in this region.

Under steady flow conditions, these large-scale shear-driven vortices dominate the rate of vertical exchange in both terrestrial (Thomas and Foken, 2007) and aquatic (Ghisalberti and Nepf, 2005) canopies. In wave-dominated flows, the generation of KH-vortices is dependent upon exceedance of threshold values of the Keulegan-Carpenter number $(K C)$ and Reynolds number $(R e)$. By taking $U_{\infty}$ (the amplitude of the above-canopy orbital velocity) and $A_{\infty}$ (the amplitude of the above-canopy horizontal excursion) as the characteristic velocity and length scales, respectively, $R e=U_{\infty} A_{\infty} / v$ (with $v$ the kinematic fluid viscosity) and $K C$ is given by:

$$
K C=\frac{U_{\infty} T}{L_{D}},
$$

where $T$ is the wave period and $L_{D}$ is the canopy drag length scale which is a measure of canopy flow resistance, defined as

$$
L_{D}=\frac{1-\lambda_{P}}{C_{D} a},
$$

(Coceal and Belcher, 2004) where $\lambda_{P}$ is the solid fraction of the canopy, $a$ the canopy frontal area per unit volume and $C_{D}$ the bulk drag coefficient of the canopy. KH-vortices are only generated at the top of the canopy when $K C$ is sufficiently large, i.e., $K C>5$ (and $R e>1000$ ) (Ghisalberti and Schlosser, 2013).

Although shear-driven vortices dominate vertical exchange in steady flows, mixing in coastal canopies is dictated by a coupled contribution of both shear- and wake-driven mixing (Abdolahpour et al., 2017a). Wake turbulence generated behind each canopy element increases in strength with the in-canopy velocity (Nepf, 1999; Abdolahpour et al., 2017a). The relative significance of wake mixing in wavedominated flows can be attributed largely to the increased in-canopy velocity under waves, relative to the corresponding steady flow (Lowe et al., 
2005). Thus, while the incorporation of canopy flexibility adds complexity to physical and numerical modeling efforts, the oscillation and timevarying drag of flexible canopies may significantly impact their hydrodynamic influence. Here, identical model rigid and flexible canopies (in terms of frontal area and maximum canopy height) were constructed so that flexibility was the only difference between the two systems. By subjecting model canopies to the same wave conditions, the impact of canopy flexibility on the structure of the oscillatory and turbulent flow, as well as on rates of vertical mixing, can be quantified.

\section{Methods}

\section{Design of flexible model vegetation}

Model plants were designed to mimic the behavior of the seagrass $P$. australis through matching the two dimensionless ratios of the forces that define plant motion, i.e. drag $\left(F_{D}\right)$, buoyancy $\left(F_{B}\right)$ and rigidity $\left(F_{R}\right)$. The first ratio $(B)$ is that of the buoyancy force to rigidity, expressed for rectangular blades as:

$$
B=\frac{F_{B}}{F_{R}}=\frac{12\left(\rho_{w}-\rho_{s}\right) g l^{3}}{E t^{2}}
$$

(Luhar and Nepf, 2011), where $\rho_{w}, \rho_{s}, l, t$ and $E$ are the density of water, the density of seagrass, the blade length, the blade thickness and the modulus of elasticity of the blade, respectively. The second dimensionless ratio, the Cauchy number $(\mathrm{Ca})$, is defined as the ratio of the drag to rigidity, and is expressed for rectangular blades as:

$$
C a=\frac{F_{D}}{F_{R}}=\frac{6 \rho_{w} C_{D} l^{3}\left(U_{c}\right)^{2}}{E t^{3}}(4)
$$


(Ghisalberti and Nepf, 2002; Luhar and Nepf, 2011), where $U_{c}$ is the amplitude of the in-canopy orbital velocity. The Cauchy number sets the plant posture; at high $\mathrm{Ca}$, the hydrodynamic forcing is large enough to overcome blade stiffness and buoyancy, and the blade more readily adjusts its posture in response to the flow (Luhar and Nepf, 2016).

To estimate values of $B$ for $P$. australis, samples were taken from two sites (Cockburn Sound and Shoalwater Bay, Western Australia). This seagrass has two (or three) differently-sized blades; the response of the longest blade was taken as the basis of comparison between real and model plants here. Average values and ranges of the length, width $(b)$, thickness and mass $(m)$ of the longest blade are presented in Table 1 . It was assumed that the blade had a constant width and thickness over its length, which resulted in an estimated seagrass density of $\rho_{s}=860 \pm 78$ $\mathrm{kg} / \mathrm{m}^{3}$. As there are no accurate estimates of $E$ for P. australis blades, it was assumed to be similar to that of Z. marina and P. Oceanica (structurally and morphologically similar species), $E=0.4-2.4 \mathrm{GPa}$ (Folkard, 2005; Luhar and Nepf, 2011). This process results in a rough estimate of $B \sim O(10)$ for $P$. australis.

Trial model blades with values of $B$ ranging from 7 - 50 (spanning the rough field estimate) were cut from Low Density Polyethylene (LDPE). Values of $B$ were varied by varying the blade height and thickness. Model blades and a real $P$. australis blade, were subjected to a wave with $U_{\infty}=28 \mathrm{~cm} / \mathrm{s}$ and $T=5 \mathrm{~s}$ to visually determine the most realistic blade motion (Figure 1a). The blade with $B \approx 12$ (with $30 \mathrm{~cm}$ length, $1 \mathrm{~cm}$ width and $300 \mu \mathrm{m}$ thickness, Figure 1a) exhibited the most realistic behavior across all wave phases. In addition to a similar oscillatory motion, this model plant displayed an identical pronation to the real plant, suggesting that the Cauchy number was also equivalent between the real and model plant. Importantly, the other model blade with similar value of $B(B \approx 11)$, which was deliberately designed to investigate the impact of $C a$, did not behave in a similar fashion to the real blade across all wave phases, despite having a comparable value of $B$ (but different $C a$ ). The blade with $B=12$ was therefore chosen as the model design. This resulted in a model plant that displayed very similar motion to real $P$. australis across all phases of the surface waves (figures $1 \mathrm{~b}-\mathrm{g}$ ). This similarity of behavior ensured that the hydrodynamic impacts of $P$. australis flexibility would be accurately recreated in the laboratory.

This article is protected by copyright. All rights reserved. 
Plant oscillation will result in a reduced drag compared to a rigid, upright element (Vogel, 1984). Definition of an effective canopy height $l_{e}$ (equivalent to the length of a rigid, vertical blade that generates the same horizontal drag as the flexible blade of length $l$ (Luhar and Nepf, 2016)) is a convenient way to describe the reduction in hydrodynamic drag due to flexibility. Under wave action, if $C a>O(1)$ and $C a>O(B)$, the blade configuration is determined by the relative magnitude of the blade length to the wave orbital excursion, $L$ ( $\left.=l / A_{\infty}\right)$ (Luhar and Nepf, 2011). Under extreme pronation (i.e. when $L \ll 1$ ), the steady flow reconfiguration model holds, which suggests that the effective height scales as

$$
l_{e} / l \sim C a^{-1 / 3}, \quad L \square 1
$$

with no dependence on $L$ (Luhar and Nepf, 2016). However, for small excursions (i.e., $L \gg 1$ ), the pronation is governed by $L$, such that

$$
l_{e} / l \sim(C a L)^{-1 / 4}, \quad L \square 1
$$

\section{Construction of rigid and flexible canopies}

Rigid and flexible model canopies with identical heights and frontal areas were constructed such that flexibility was the only difference between the two types of canopy. Rigid model plants with a canopy height of $h_{c}=30 \mathrm{~cm}$ were made of $d=6.4 \mathrm{~mm}$ birch dowels hammered into perforated PVC boards. Three rigid canopies, with dimensionless frontal areas $a d=0.016,0.063$ and 0.131 (symbolized as RL, RM and RH hereafter) were employed (Table 2).

To mimic the morphology of $P$. australis, each flexible model plant consisted of two differently-sized blades (with heights 15 and $30 \mathrm{~cm}$ ) attached to a $3 \mathrm{~cm}$ wooden dowel at the base (Figures 2a and b). Thus, the canopy frontal area varied over depth. Flexible canopies were described through their vertically-averaged dimensionless frontal areas $a b_{e f f}=0.015,0.064$ and 0.145 (symbolized as FL, FM and FH hereafter; see Table 2 and vertical dashed line in Figure 2c). These dimensionless frontal areas were chosen to be equivalent (to within 10\%) to those of the 
rigid canopies. Note that $a b_{e f f}$ is the dimensionless frontal area of the canopy based on the full blade height and width. The ranges of $a d$ ( 0.016 $0.131)$ and $a b_{\text {eff }}(0.015-0.145)$ employed here span typical densities of coastal canopies (Luhar et al., 2010).

\section{Experimental setup}

Experiments were carried out in a $50 \mathrm{~m}$ long, $1.2 \mathrm{~m}$ deep, and $1.2 \mathrm{~m}$ wide wave flume. The wave flume was equipped with a programmable piston-type wave generator located approximately in the middle of the flume. Wooden beaches of slope 1:10 (covered by anti-fatigue rubber mats) were constructed at both ends of the tank to reduce the reflection coefficient of the flume to less than $2-10 \%$, for the wave conditiones typical of this study (see Abdolahpour et al., 2017b, for details). The still water depth $(h=0.76 \mathrm{~m})$ was kept constant throughout the experiments. The canopy length $\left(L_{c}\right)$ was varied inversely with canopy density (ranging from 3-9 m, Table 2).

Each canopy was exposed to nineteen flow conditions by varying the wave period $\left(T=5-9 \mathrm{~s}\right.$ ) and above-canopy RMS velocity ( $U_{\infty}^{\text {rms }}=3.0-22$ $\mathrm{cm} / \mathrm{s}$ ) (Table 3). All generated waves were shallow-water waves with $k h<0.35$ (where $k$ is the wave number), typical of coastal canopies (Koch et al., 2006). The range of wave and canopy conditions selected here resulted in $R e>1000$ and $1<K C<40$ (Table 3). This range, in addition to spanning typical field ranges of marine canopies (Luhar et al., 2008; Ghisalberti and Schlosser, 2013), allowed investigation of cases with and without the generation of KH-vortices.

\section{Velocity and turbulence measurements}

Here, $x$ is defined as the direction of wave propagation, $y$ as the lateral direction and $z$ as the vertical direction (positive upward), with $z=0$ at the bed. Velocity components along $x, y$ and $z$ directions are denoted (respectively) by $u, v$ and $w$.

Vertical velocity profiles were taken in the middle of the canopy using a SonTek Acoustic Doppler Velocimeter (ADV) at a sampling frequency of $25 \mathrm{~Hz}$. In rigid canopies, data points were spaced every $2 \mathrm{~cm}$. In the flexible canopies, vertical measurements were taken every $1 \mathrm{~cm}(\mathrm{starting}$ 
at $2 \mathrm{~cm}$ from the bed) for $z, 16 \mathrm{~cm}$, and continued every $2 \mathrm{~cm}$ for $z>16 \mathrm{~cm}$. This allowed focus on the near-bed region, where the drag, turbulence and shear were expected to be greatest (Lowe et al., 2005; Abdolahpour et al., 2017a,b).

In flexible canopies, blade motion through the wave cycle can interfere with the sampling volume of the ADV. Rather than trimming or removing a requisite amount of blades, which has been shown to have a non-trivial impact on the magnitude of RMS velocities near the top of the canopy (by up to 30\%), flexible canopies were not modified to allow ADV access. Instead, ADV data that were contaminated by model blades entering the sampling volume (beam correlations $<70 \%$ ) were excluded from analysis. For rigid canopies, a sampling time of 6 minutes ( 40 - 70 wave cycles) was employed; for flexible canopies, this was increased to 10 minutes $(70-120$ wave cycles) to allow for data exclusion (see Abdolahpour et al., 2017b, for details of data exclusion).

The three-dimensional instantaneous flow velocities were decomposed into a steady component associated with the time-independent mean motion, an unsteady component associated with the oscillatory flow and a turbulent component (indicated by an overbar, a tilde and a prime, respectively). That is, the velocity field in the direction of wave propagation, for example, can be represented as

$$
u(z, t)=\bar{u}(z)+\tilde{u}(z, t)+u^{\prime}(z, t)
$$

At any given height, the RMS of the oscillatory velocity in the direction of wave propagation, $U^{r m s}$, was calculated according to:

$$
U^{r m s}=\sqrt{\frac{1}{2 \pi} \int_{-\pi}^{\pi}(\langle u(\varphi)\rangle-\bar{u})^{2} d \varphi}
$$

with the angular brackets representing the phase average over several wave periods. Here, zero phase occurs under the wave crest and $-\pi$ and $\pi$ under the wave trough. The velocity attenuation within the canopy, $\Delta U$, was calculated as:

$$
\Delta U=U_{\infty}^{r m s}-U_{c}^{r m s}
$$


(Figure 5). For flexible canopies, the lowermost measurement of $U^{r m s}$ was discarded when estimating the mean in-canopy velocity value. This point $(\mathrm{z} \approx 2 \mathrm{~cm})$ was located within the region of the canopy occupied by stems, rather than blades.

To isolate turbulent fluctuations, the mean and wave components were removed from velocity signals by applying a sixth-order high pass filter to velocity signals after subtraction of the mean. The cut-off frequency was set to triple the wave frequency, a threshold beyond the frequencies of the primary wave and second harmonic. The time-averaged Turbulent Kinetic Energy ( $T K E$ ) was then calculated as

$$
T K E=\frac{1}{2}\left[\overline{\left(u^{\prime}\right)^{2}}+\overline{\left(v^{\prime}\right)^{2}}+\overline{\left(w^{\prime}\right)^{2}}\right]
$$

with the phase-averaged Turbulent Kinetic Energy calculated as

$$
\langle T K E\rangle=\frac{1}{2}\left(\left\langle u^{\prime}(\varphi)^{2}\right\rangle+\left\langle v^{\prime}(\varphi)^{2}\right\rangle+\left\langle w^{\prime}(\varphi)^{2}\right\rangle\right)
$$

The time- and phase-averaged Reynolds stresses, which indicates the rate of vertical mixing of horizontal momentum and provides qualitative insight into the vertical mixing of mass, were also calculated. If $u$ ' and $w$ ' are taken as positive in the shoreward and upward direction respectively, $u$ ' $w^{\prime}$ will then be expected to become negative in the middle of the shear layer, representing the downward transport of high momentum from the overlying flow. In oscillatory flows, the horizontal velocity switches sign as the flow reverses, such that $u{ }^{\prime}{ }^{\prime}$ changes sign with each wave half-cycle. Therefore, the time-averaged Reynolds stress, $\overline{u^{\prime} w^{\prime}}$, tends to zero (Nielsen, 1992; Lamb et al., 2004). Thus, in order to accurately quantify the total rate of downward momentum transport, $\overline{|u| w^{\prime}}$ was calculated (Ghisalberti and Schlosser, 2013) to provide a better representation of vertical mass transport.

\section{Model for vertical mixing}


Vertical mixing is characterized here by quantifying the vertical turbulent diffusivity $\left(D_{t, z}\right)$, estimated from gauging the evolution of vertical profiles of concentration $(C)$ of a dye sheet injected into the flow. When concentration is uniform in the horizontal with no vertical advection, the solution to mass transport equation for an instantaneous release of mass $M$ of a tracer at point $z=z_{0}$ at time $t=0$ is given by

$$
C(z, t)=\left(\frac{M}{A_{x y} \sqrt{4 \pi D_{t, z} t}}\right)\left(\exp \frac{-\left(z-z_{0}\right)^{2}}{4 D_{t, z} t}\right)
$$

where $A_{x y}$ is the $x-y$ planar area. An image source must be added to this solution if the tracer reaches the no-flux boundaries (either the bed or the water surface). The concentration distribution along any vertical line through the tracer cloud will have a Gaussian distribution, with a variance $\left(\sigma^{2}\right)$ that increases linearly with time, such that

$$
\frac{d \sigma^{2}}{d t}=2 D_{t, z}
$$

(Fischer, 1972). Consequently, measurement of the rate of change of variance of the vertical concentration distribution allows estimation of the vertical turbulent diffusivity (Abdolahpour et al., 2017a).

\section{Measurement of vertical turbulent diffusivity}

A two-line dye injection system was used to create a sheet of Rhodamine WT dye that was horizontally uniform (Figure 3a, Abdolahpour et al. (2017a)), a condition of employing eq. (12). Dye injection lasted one to four wave cycles, creating a dye sheet with a length approximately equal to the total particle excursion (ranged from $15-60 \mathrm{~cm}$ ).

For rigid canopies, dye injection was at the top of the canopy (i.e., $z_{0}=h_{c}$ in eq. 12), where the velocity shear was expected to be greatest. Five submersible fluorometers (sampling frequency $15 \mathrm{~Hz}$ ) were arranged vertically (every $15 \mathrm{~cm}$, Figure 3a), centered around the canopy top such 
that the fluorometer in the middle of the array was coincident with the top of the canopy and the injection system (Abdolahpour et al., 2017a). These fluorometers were used to determine the instantaneous vertical concentration distribution of the dye. For flexible canopies since there is no well-defined canopy top, a bed injection (i.e., $z_{0}=0$ in eq. 12) was employed, with the five fluorometers situated every $7.5 \mathrm{~cm}$ from the bed (Figure 3b).

The simultaneous measurements of concentration at these five vertical locations were used to construct instantaneous estimates of the variance of the concentration distribution $\left(\sigma^{2}-t\right)$ and, ultimately, $D_{t, z}$ in each experiment (eq. 13$)$.

The cloud variance increased linearly with time from the time of injection and the slope of the linear portion of the $\sigma^{2}-t$ curve was used to calculate $D_{t, z}$ (eq. 13) (see Abdolahpour et al., 2017a; Abdolahpour, 2017). The average values of $D_{t, z}$ for each run (over 3 - 8 replicates) are shown in Table 3.

The impact of injection location on the estimation of $D_{t, z}$ was investigated for 12 runs in the dense flexible canopy $\left(a b_{e f f}=0.145\right)$ through injection at $z=0$ (i.e. at the bed) vs. injection at $z=7 \mathrm{~cm}$. The injection at $z=7 \mathrm{~cm}$ fell both within the shear layer (for 5 runs) and outside it (for 7 runs). There was no observed correlation between the injection location and estimated diffusivity; the average ratio of the diffusivities obtained from these two methods was $\approx 0.94$ and the average discrepancy was $<15 \%$ which was deemed to be negligible compared to the significant variation (by an order of magnitude) due to changes in canopy and wave characteristics (Table 3). Thus, this method allows evaluation of a single diffusivity that describes mass transport into and out of the canopy.

\section{Results}

\section{Effective canopy height}


Predictive capability for the effective height of flexible canopies in oscillatory flows is important for an accurate description of flow characteristics in real coastal canopies with significant flexibility. Accordingly, the applicability of equations (5) and (6) in predicting this effective height was examined for the range of $B$ and $C a$ values tested here. As the drag force (which is normally used to determine the effective height (Luhar and Nepf, 2016)) was not measured in this study, a hydrodynamic definition of the effective height was employed. That is, the height of the center of the shear layer $l_{s l}$ (i.e., the height of the inflection point in the vertical profile of $U^{r m s}$ ) was adopted as a measure of the effective height of flexible canopies. For all runs, $C a>O(1)$ and $C a>O(B)$ (conditions of applying equations (5) and (6)), with $L$ ranging from 0.7 to 3.5. Runs with $A_{\infty} / L_{D} \wedge 1$ (Table 3) were characterized by small horizontal excursions and hence negligible velocity attenuation (Zeller et al., 2015). These runs were discarded in this analysis due to the absence of clear inflection points in the vertical profiles of $U^{r m s}$.

For both $L<1$ and $L>1$, our observations are consistent with equations (5) and (6), respectively (Figures 4a and b). The effective canopy height can be as low as $20 \%$ of the maximum height (i.e., $l_{s} / l \sim 0.2$ ), indicating that the shear layer of flexible canopies can be drastically shifted towards the bed.

\section{Time-averaged oscillatory and turbulent flow fields}

Vertical profiles of $U^{r m s}$ in both rigid and flexible canopies exhibit significant velocity gradients whose strength increases with canopy density (Figure 5). The region of shear is centered around the canopy top in rigid canopies ( $z=30 \mathrm{~cm}$, Figure 5a) and centered around the effective height in flexible canopies $\left(\mathrm{z}=l_{s l}<30 \mathrm{~cm}\right.$, Figure $5 \mathrm{~b}$ ). In flexible canopies, the upper region of the canopy oscillates throughout the wave cycle, thus reducing the relative velocity between the flow and blades and, ultimately, the drag exerted by the upper region. Therefore, the drag is mainly due to the lower region of the canopy which stays approximately upright and motionless. The time-varying canopy height may explain the thicker shear layer generated in flexible canopies (gray bands in Figure 5a and b).

Flexible canopies exhibited a diminished velocity attenuation compared to the rigid analogues. On average, there is a $65 \%$ reduction in velocity attenuation in flexible canopies compared to the corresponding rigid canopies when subjected to the same wave conditions (Figure 6). This is 
due to the ability of flexible canopies to adjust their shape and posture in response to the flow, thus reducing the relative velocity between the blade and the ambient flow. The diminished drag that results from this greatly reduces velocity attenuation within the canopy.

Vertical profiles of the time-averaged Reynolds stress $\overline{|u|^{\prime}}$ for $K C>5$ (cases with vortex generation) are presented in Figure 7a. A region of strong momentum transfer, which increases with canopy density (results not shown), is observed within the shear layer; i.e. near the canopy top in rigid canopies and near the effective height in flexible canopies (Figure 7a). This is due to the enhanced velocity shear and the resultant KHvortices in these regions. It is noteworthy that the reduced average height of flexible canopies shifts the canopy shear layer towards the bed, resulting in a greatly enhanced level of near-bed turbulence. Additionally, the magnitude of $\overline{|u|^{\prime}}$ is diminished in flexible canopies such that the peak value of $\overline{|u|^{\prime}}$ is approximately 50\% less than in the corresponding rigid canopy. This is due to the weakened velocity gradients in flexible canopies (Figures 5 and 6), which result in diminished momentum transfer.

The time-averaged T KE (for the same runs as in Figure 5) provides further understanding of the turbulent flow structure in rigid and flexible canopies (Figure 7a and b). In both cases, levels of $T K E$ are greater within the canopy than above it, due to the presence of wake turbulence in this region. Moreover, the magnitude of $T K E$ is significantly greater in flexible canopies due to the higher in-canopy velocity in these systems (Figure 5); the greater the in-canopy velocity, the stronger the generation of wake turbulence.

There is a noticeable peak in $T K E$ within the shear layer in the flexible canopy, with a magnitude more than double that in the corresponding rigid canopy. This suggests the generation of strong turbulent fluctuations by the oscillatory motion of flexible canopies, as seen by Koch and Gust (1999). In combination with the reduced canopy height, flexible canopies can thus introduce significant turbulence to the near-bed region.

\section{Instantaneous oscillatory flow and turbulence}


The phase-dependent structure of the oscillatory flow and turbulence for runs with $K C>5$ is demonstrated in Figure 8 . Due to the canopy resistance, the in-canopy velocity leads the above-canopy velocity by a phase angle of up to $\pi / 6$ in rigid canopies (Figure $8 a$ ). This phase lag is less pronounced in flexible canopies due to the reduced drag in these environments (Figure 8b).

The vertical mixing of momentum, as indicated by $\left\langle|u|^{\prime} w^{\prime}\right\rangle$, is highly phase-dependent. Its magnitude increases under the wave crest $(\varphi=0)$ and trough $(\varphi= \pm \pi)$ in both rigid (Figure 8c) and flexible (Figure 8d) canopies. The enhancement of $\left\langle|u|^{\prime} w\right\rangle$ is, however, much stronger under the wave crest. This is potentially due to the strong mean current in the direction of wave propagation at the canopy top (Abdolahpour et al., 2017b). The higher velocity induced by this current increases the shear under the wave crest and ultimately the momentum transfer. The region of upward momentum transfer (i.e. positive $\left\langle|u|^{\prime} w^{\prime}\right\rangle$ ) immediately above the rigid canopy is due to the strong overshoot occurring in this area. This overshoot (well-defined in Figure 5a), causes high-speed fluid particles to be transported upward into the (comparatively) low-speed environments above.

The phase-averaged $T K E$ also reveals a strong dependence on the wave phase, with $\langle T K E\rangle$ increasing under the wave crest and trough (Figure 8e). This phase dependence is more pronounced in the rigid canopy; i.e. in the flexible canopy, the augmentation of turbulent kinetic energy is not limited to under the wave crest and trough; it is indeed distributed across all wave phases. This is again suggestive of the generation of turbulence due to the motion of flexible canopies and serves to further enhance levels of turbulence near the bed.

\section{Turbulent scales}

Spectra of vertical turbulent velocity, $S_{w^{\prime} w^{\prime}}$ were analyzed to demonstrate the change in the turbulent structure in rigid and flexible canopies as the $K C>5$ threshold (for the generation of $\mathrm{KH}$-vortices) is exceeded. Spectral densities in two frequency bands were compared, namely those corresponding to the natural frequency of $\mathrm{KH}$ and wake vortices. The frequency of $\mathrm{KH}$ instability $\left(f_{\mathrm{KH}}\right)$ in steady flows was used here to approximate the former; i.e., 


$$
f_{K H}=0.032\left(\frac{\bar{U}_{s l}}{\theta}\right)
$$

(Ghisalberti and Nepf, 2002), where $\bar{U}_{s l}$ is the mean of the far above- and within-canopy velocities and $\theta \approx t_{s l} / 7$ is the momentum thickness of the shear layer, where $t_{s l}$ is the thickness of the shear layer; therefore, $f_{K H} \approx 0.2 \bar{U}_{s l} / t_{s l}$. In wave-dominated flows, where there is no single measure of velocity, evaluation of $\bar{U}_{s l}$ is tricky. Here, averages of above- and within-canopy values of both $U^{r m s}$ and $U$ (the amplitude of the orbital velocity) were used to estimate minimum and maximum bounds for $\bar{U}_{\text {sl }}$, and thus an approximate frequency range for KH-vortices in oscillatory flows.

Insight into the frequency of wake turbulence $\left(f_{w}\right)$ can also be gained from steady flows in which

$$
f_{w} \approx 0.2 \frac{w}{\bar{U}_{c}}
$$

(Norberg, 2001), where $w=d$ for cylinders, $w=b$ for blades, and $\bar{U}_{c}$ is the time-averaged in-canopy velocity. Again, definition of a singular incanopy velocity for wave-dominated flows is difficult. Thus, a frequency range was also estimated for wake turbulence, taking both $U_{c}^{\text {rms }}$ and $U_{c}$ as measures of the in-canopy velocity.

Spectra of vertical turbulent velocities (1) within the canopy (where wakes are dominant, $z=0.5 l_{s l}$ ), (2) within the shear layer (where KHvortices are dominant, $z=l_{s l}$ ) and (3) well above the canopy (outside of the impact of wake and shear layer vortices, $z . .2 l_{s l}$ ) are presented in Figure 9. The estimated frequency ranges for KH-vortices and wake turbulence are shown in light gray and dark gray bands, respectively. For rigid canopies, the energy spectrum within the canopy demonstrates similar characteristics to that within the shear layer across all frequencies when $K C<5$ (Figure 9a). However, there is greatly enhanced turbulent energy within the shear layer at lower frequencies (i.e., $0.4<f<0.9 \mathrm{~Hz}$ ) when $K C>5$ (Figure 9c). The frequencies of elevated energy (light gray arrow in Figure 9c) falls within the frequency band of KH instability, 
indicative of the generation of shear-driven vortices near the canopy top. As in rigid canopies, there is no significant vertical variation in flexible canopy spectra in the KH-frequency band when $K C<5$ (Figure $9 \mathrm{~b}$ ). However, a clear augmentation of $S_{w^{\prime} w^{\prime}}$ is observed in this band within the shear layer when $K C>5$ (Figure 9d). A direct comparison of spectra from rigid and flexible canopies reveals that this augmentation of energy is diminished in flexible canopies (indicated by light gray arrows in Figures 9c and d). This is consistent with the results observed in Figures 5, 7 and 8 where the weaker velocity gradients generated in flexible canopies result in weaker shear layer turbulence.

The contribution of wake-scale turbulence at higher frequencies (black arrows in Figure 9) to the total turbulent energy within the canopy is significant; even at high $K C$; it is comparable to the contribution of shear layer turbulence. This supports the observation of wake turbulence playing a significant role in vertical mixing in oscillatory canopy flows, particularly when large scale $\mathrm{KH}$-vortices are absent (i.e. $\mathrm{KC}<5$ ) (Abdolahpour et al., 2017a).

\section{The impact of flexibility on vertical mixing}

Vertical mixing in rigid coastal canopies is characterized by a coupled contribution of both shear- and wake-driven mixing and can be quantified by

$$
D_{t, z}=0.043 \Delta U L_{D}+0.58 \sqrt[3]{\frac{w}{L_{D}}} U_{c}^{r m s} w
$$

(Abdolahpour et al., 2017a). The first term on the right hand side of (16) represents the contribution of shear-driven mixing and the second term the contribution of wake-driven mixing. In flexible canopies, shear-driven mixing is expected to decrease due to the diminished velocity attenuation and weakened shear layer vortices (Figures 6, 7 and 8). Conversely, the diminished flow attenuation leads to an increase in the incanopy velocity, to which the wake-driven mixing is directly proportional (Abdolahpour et al., 2017a). Thus, in contrast to shear layer mixing, wake-driven mixing increases in flexible canopies. 
The applicability of eq. (16) in describing rates of vertical mixing in flexible canopies is depicted in Figure 10a. Although a reasonably good agreement is observed between measured and predicted values of $D_{t, z}$ (with $R^{2}=0.8$ ), accurate estimation of all parameters in eq. (16) may not be feasible in real coastal canopies. The net effect of those counteractive mechanisms, i.e. reduction of shear-driven mixing and augmentation of wake-driven mixing, is a significant reduction (on average 35\%) in the vertical turbulent diffusivity in flexible canopies (Figure 10b) which is independent of wave and canopy conditions and does not show any meaningful dependence on $K C$.

\section{Discussion}

While it is often advantageous to create models without the complexity of real systems, the results obtained here highlight the limitations of using rigid elements to simulate aquatic canopies. In particular, flexibility is observed to (1) shift the canopy shear layer towards the bed, (2) diminish velocity attenuation within the canopy (3) weaken momentum transfer in the shear layer and, ultimately, (4) reduce vertical mixing. Accordingly, it is expected that flexibility will impact important ecological and biological processes within coastal canopies. For example, the downward shift of shear layer turbulence (Figure 7) could enhance sediment resuspension (Hansen and Reidenbach, 2013; Ros et al., 2014) and thus turbidity (Duarte, 1995; Wang et al., 2010). The enhancement of resuspension also has implications for near-bed processes such as sediment transport (Nelson et al., 1995) and material exchange across the sediment-water interface (Hondzo, 1998; Reidenbach et al., 2010).

The elevated in-canopy velocity in flexible canopies is likely to increase fluxes of dissolved and particulate material to canopy elements (e.g. Lei and Nepf, 2016). Previous studies have shown that the capture of suspended particles with canopy elements (Ackerman, 2002; Palmer et al., 2004; Shimeta, 2009) increases with increasing velocity. Thus, processes such as pollination (Smith and Walker, 2002; Verduin et al., 2002), rates of suspension feeding (Humphries, 2009) and larval settlement (Harvey et al., 1995) may also be increased as a result of canopy flexibility. In addition to these, the motion of flexible blades through the water column can increase the rate of particle capture by increasing the likelihood of blade exposure to the more nutrient-rich environments as shown by, e.g., Grizzle et al. (1996). 
The lowered rates of vertical mixing in flexible canopies will reduce the flux of dissolved and particulate material (e.g. pollen, seeds, sediment, oxygen and pollutants) across the canopy boundaries. This increases residence time within the canopy, defined as the region from the bed to the maximum height of the canopy, which will significantly influence the retention of ecologically-significant materials. For example, by regulating rates of seed and pollen dispersal, a greater residence time can play an essential role in the distribution and abundance of plants across a landscape, the spread of existing populations and the potential for new population formation (Harwell and Orth, 2002; Nathan et al., 2008). In combination with the enhanced resuspension, the diminished vertical efflux from the canopy can serve to increase the in-canopy turbidity. The ecological implications of the greater residence time in flexible canopies are complex, since some processes may be enhanced by longer residence times (e.g. particle trapping, turbidity and nutrient uptake), while others may be reduced (e.g. resupply of nutrients through flushing), with the net effect on ecosystem function difficult to predict (Harvey et al., 2003; Hendriks et al., 2008). In any case, using simplified rigid model elements will clearly underestimate the residence time in real systems where flexibility is a salient feature of the canopy.

The results obtained here are in excellent agreement with existing field observations of vertical turbulent diffusivities of $O\left(1 \mathrm{~cm}^{2} / \mathrm{s}\right)$ within a canopy of Z. marina (Ackerman and Okubo, 1993; Ackerman, 2002). Thus, with a simple correction factor (i.e. a 35\% reduction in mixing), the predictive model for vertical mixing proposed by Abdolahpour et al. (2017a) can conveniently be employed in cases where an accurate estimation of wave and canopy properties is not practical. The validity of eq. (16) in describing vertical mixing in flexible canopies is a fundamentally-important result as it enables an enhanced capability to understand and predict the transport and residence time of dissolved and particulate materials (such as nutrients, contaminants and suspended sediment) in real coastal canopies.

It is important to note that seagrass blades often support large biomasses of epiphytic organisms (Borowitzka et al., 2007) which add significant structural complexity to the canopy. In addition to this, real canopies are frequently composed of a mixture of species with vertical morphological variation. While creating model plants with differently-sized flexible blades allowed a more faithful examination of depth-varying canopy drag on the hydrodynamics of oscillatory flow, the model blades used here were entirely uniform and may not represent the complexity of many real systems. Moreover, this study provides a representative value for the residence time in canopies exposed to idealized sinusoidal 
waves with a single peak in the wave spectrum. Characterization of the hydrodynamic influence of multi-specific canopies in real coastal systems is an important next step in assessing the impact of canopy structure on flow and mixing.

Acknowledgments M. Abdolahpour gratefully acknowledges the support of a Collaborative Research Network scholarship (CRN) and a Postgraduate Research Scholarship granted by Edith Cowan University (ECUPRS). This project was funded by a grant from the Australian

Department of Innovation, Industry, Science and Research’s Collaborative Research Network Scheme (Grant CRN2011:05). The authors would also like to thank Brett Branco for providing the fluorometers used in this study.

\section{References}

Abdolahpour, M. (2017), Residence time in coastal canopies, Ph.D. thesis, The University of Western Australia and Edith Cowan University.

Abdolahpour, M., M. Ghisalberti, P. Lavery, and K. McMahon (2017a), Vertical mixing in coastal canopies, Limnol. Oceanogr., 62(1), 26-42, doi:10. 1002/lno.10368.

Abdolahpour, M., M. Hambleton, and M. Ghisalberti (2017b), The wave-driven current in coastal canopies, J. Geophys. Res. Ocean., (122), doi:10.1002/2016JC012446.

Ackerman, J., and A. Okubo (1993), Reduced mixing in a marine macrophyte canopy, Funct. Ecol., pp. 305-309.

Ackerman, J. D. (2002), Diffusivity in a marine macrophyte canopy: implications for submarine pollination and dispersal, Am. J. Bot., 89(7), 1119-1127.

Albayrak, I., V. Nikora, O. Miler, and M. OHare (2012), Flow-plant interactions at a leaf scale: effects of leaf shape, serration, roughness and flexural rigidity, Aquat. Sci., 74(2), 267-286. 
Barbier, E. B., S. D. Hacker, C. Kennedy, E. W. Koch, A. C. Stier, and B. R. Silliman (2011), The value of estuarine and coastal ecosystem services, Ecol. Monogr., 81(2), 169-193.

Borowitzka, M. A., P. S. Lavery, and M. van Keulen (2007), Epiphytes of seagrasses, Springer, Netherlands.

Bouma, T., M. De Vries, E. Low, G. Peralta, I. v. Tánczos, J. van de Koppel, and P. J. Herman (2005), Trade-offs related to ecosystem engineering: A case study on stiffness of emerging macrophytes, Ecology, 86(8), 2187-2199.

Coceal, O., and S. Belcher (2004), A canopy model of mean winds through urban areas, Quart. J. Roy. Meteor. Soc., 130(599), $1349-1372$.

Connolly, R. M., J. S. Hindell, and D. Gorman (2005), Seagrass and epiphytic algae support nutrition of a fisheries species, Sillago schomburgkii, in adjacent intertidal habitats, Mar. Ecol. Prog. Ser., 286, 69-79.

Denny, M., and L. Roberson (2002), Blade motion and nutrient flux to the kelp, Eisenia arborea, Biol. Bull., 1, 203.

Duarte, C. M. (1995), Submerged aquatic vegetation in relation to different nutrient regimes, Ophelia, 41(1), 87-112.

Duarte, C. M. (2002), The future of seagrass meadows, Environ. Conserv., 29(02), 192-206.

Fischer, H. B. (1972), Mass transport mechanisms in partially stratified estuaries, J. Fluid Mech., 53(4), 671-687.

Folkard, A. M. (2005), Hydrodynamics of model posidonia oceanica patches in shallow water, Limnol. Oceanogr., 50(5), 1592-1600.

Fonseca, M. S., and J. A. Cahalan (1992), A preliminary evaluation of wave attenuation by four species of seagrass, Estuar. Coast. Shelf. Sci., 35(6), 565-576. 
Fonseca, M. S., W. J. Kenworthy, and G. W. Thayer (1992), Seagrass beds: nursery for coastal species, in Stemming the Tide of Coastal Fish Habitat Loss. Marine Recreational Fisheries Symposium, Baltimore, MD (USA), pp. 141-147, National Coalition for Marine Conservation, Savannah, GA (USA).

Gacia, E., T. Granata, and C. Duarte (1999), An approach to measurement of particle flux and sediment retention within seagrass (Posidonia oceanica) meadows, Aquat. Bot., 65(1), 255-268.

Ghisalberti, M., and H. Nepf (2005), Mass transport in vegetated shear flows, Environ. Fluid Mech., 5(6), 527-551.

Ghisalberti, M., and H. Nepf (2006), The structure of the shear layer in flows over rigid and flexible canopies, Environ. Fluid Mech., 6(3), 277301.

Ghisalberti, M., and H. Nepf (2009), Shallow flows over a permeable medium: the hydrodynamics of submerged aquatic canopies, Transp. Porous Media., 78(2), 309-326.

Ghisalberti, M., and H. M. Nepf (2002), Mixing layers and coherent structures in vegetated aquatic flows, J. Geophys. Res., $107(\mathrm{C} 2)$, 3011.

Ghisalberti, M., and T. Schlosser (2013), Vortex generation in oscillatory canopy flow, J. Geophys. Res. Ocean., 118(3), $1534-1542$.

Granata, T., T. Serra, J. Colomer, X. Casamitjana, C. Duarte, E. Gacia, and J. Petersen (2001), Flow and particle distributions in a nearshore seagrass meadow before and after a storm, Mar. Ecol. Prog. Ser., 218, 95-106.

Grizzle, R. E., F. T. Short, C. R. Newell, H. Hoven, and L. Kindblom (1996), Hydrodynamically induced synchronous waving of seagrasses:monamiand its possible effects on larval mussel settlement, J. Exp. Mar. Bio. Ecol., 206(1-2), 165-177. 
Hansen, J. C., and M. A. Reidenbach (2011), Wave and tidally driven flows in eelgrass beds and their effect on sediment suspension, Mar. Ecol. Prog. Ser., 448, 271-287.

Hansen, J. C., and M. A. Reidenbach (2013), Seasonal growth and senescence of a Zostera marina seagrass meadow alters wave-dominated flow and sediment suspension within a coastal bay, Estuar. Coast., 36(6), 1099-1114.

Harvey, J. W., M. H. Conklin, and R. S. Koelsch (2003), Predicting changes in hydrologic retention in an evolving semi-arid alluvial stream, Adv. Water Resour., 26(9), 939-950.

Harvey, M., E. Bourget, and R. G. Ingram (1995), Experimental evidence of passive accumulation of marine bivalve larvae on filamentous epibenthic structures, Limnol. Oceanogr., 40(1), 94-104.

Harwell, M. C., and R. J. Orth (2002), Long-distance dispersal potential in a marine macrophyte, Ecology, 83(12), 3319-3330.

Hendriks, I. E., T. Sintes, T. J. Bouma, and C. M. Duarte (2008), Experimental assessment and modeling evaluation of the effects of seagrass Posidonia oceanica on flow and particle trapping, Mar. Ecol. Prog. Ser., 356.

Hondzo, M. (1998), Dissolved oxygen transfer at the sediment-water interface in a turbulent flow, Water Resour. Res., 34(12), 3525-3533.

Houser, C., S. Trimble, and B. Morales (2014), Influence of blade flexibility on the drag coefficient of aquatic vegetation, Estuar. Coast., pp. 19.

Huang, I., J. Rominger, and H. Nepf (2011), The motion of kelp blades and the surface renewal model, Limnol. Oceanogr., 56(4), $1453-1462$.

Humphries, S. (2009), Filter feeders and plankton increase particle encounter rates through flow regime control, Proc. Natl. Acad. Sci. U.S.A, 106(19), 7882-7887. 
Hurd, C. L. (2000), Water motion, marine macroalgal physiology, and production, J. Phycol., 36(3), 453-472.

Kobayashi, N., A. W. Raichle, and T. Asano (1993), Wave attenuation by vegetation, J. Waterw. Port Coast. Ocean Eng., 119(1), 30-48.

Koch, E. W., and G. Gust (1999), Water flow in tide-and wave-dominated beds of the seagrass thalassia testudinum, Mar. Ecol. Prog. Ser., 184(1), 63-72.

Koch, E. W., L. P. Sanford, S.-N. Chen, D. J. Shafer, and J. M. Smith (2006), Waves in seagrass systems: review and technical recommendations, Tech. rep., DTIC Document.

Koehl, M., W. Silk, H. Liang, and L. Mahadevan (2008), How kelp produce blade shapes suited to different flow regimes: a new wrinkle, Integr. Comp. Biol., 48(6), 834-851.

Lamb, M. P., E. D’Asaro, and J. D. Parsons (2004), Turbulent structure of high-density suspensions formed under waves, J. Geophys. Res. Ocean., 109(C12).

Larkum, A. W., R. R. J. Orth, and C. M. Duarte (2006), Seagrasses: biology, ecology, and conservation, Springer, Dordrecht.

Lei, J., and H. Nepf (2016), Impact of current speed on mass flux to a model flexible seagrass blade, J. Geophys. Res. Ocean., 121(7), 47634776.

Lowe, R. J., J. R. Koseff, and S. G. Monismith (2005), Oscillatory flow through submerged canopies: 1. velocity structure, J. Geophys. Res. Ocean., 110(C10).

Luhar, M., and H. Nepf (2016), Wave-induced dynamics of flexible blades, J. Fluid Struct., 61, $20-41$. 
Luhar, M., and H. M. Nepf (2011), Flow-induced reconfiguration of buoyant and flexible aquatic vegetation, Limnol. Oceanogr., 56(6), 20032017.

Luhar, M., J. Rominger, and H. Nepf (2008), Interaction between flow, transport and vegetation spatial structure, Environ. Fluid Mech., 8(5-6), 423-439.

Luhar, M., S. Coutu, E. Infantes, S. Fox, and H. Nepf (2010), Wave-induced velocities inside a model seagrass bed, J. Geophys. Res. Ocean., 115(C12).

Manca, E., I. Cáceres, J. Alsina, V. Stratigaki, I. Townend, and C. Amos (2012), Wave energy and wave-induced flow reduction by full-scale model Posidonia oceanica seagrass, Cont. Shelf Res., 50, 100-116.

Mass, T., A. Genin, U. Shavit, M. Grinstein, and D. Tchernov (2010), Flow enhances photosynthesis in marine benthic autotrophs by increasing the efflux of oxygen from the organism to the water, Proc. Natl. Acad. Sci. U.S.A, 107(6), 2527-2531.

Moore, K. A. (2004), Influence of seagrasses on water quality in shallow regions of the lower chesapeake bay, J. Coast. Res., pp. $162-178$.

Nathan, R., F. M. Schurr, O. Spiegel, O. Steinitz, A. Trakhtenbrot, and A. Tsoar (2008), Mechanisms of long-distance seed dispersal, Trends Ecol. Evol., 23(11), 638-647.

Nelson, J. M., R. L. Shreve, S. R. McLean, and T. G. Drake (1995), Role of near-bed turbulence structure in bed load transport and bed form mechanics, Water Resour. Res., 31(8), 2071-2086.

Nepf, H. (1999), Drag, turbulence, and diffusion in flow through emergent vegetation, Water Resour. Res., 35(2), 479-489.

Nepf, H. M. (2012a), Flow and transport in regions with aquatic vegetation, Annu. Rev. Fluid Mech., 44, 123-142. 
Nepf, H. M. (2012b), Hydrodynamics of vegetated channels, J. Hydraul. Res., 50(3), 262-279.

Nielsen, P. (1992), Coastal bottom boundary layers and sediment transport, World Sci., Hackensack, NJ.

Nishihara, G. N., R. Terada, and H. Shimabukuro (2011), Effects of wave energy on the residence times of a fluorescent tracer in the canopy of the intertidal marine macroalgae, Sargassum fusiforme (Phaeophyceae), Phycol. Res., 59(1), 24-33.

Norberg, C. (2001), Flow around a circular cylinder: aspects of fluctuating lift, J. Fluid Struct., 15(3-4), 459-469.

Palmer, M. R., H. M. Nepf, T. J. Pettersson, and J. D. Ackerman (2004), Observations of particle capture on a cylindrical collector: Implications for particle accumulation and removal in aquatic systems, Limnol. Oceanogr., 49(1), 76-85.

Paul, M., T. Bouma, and C. Amos (2012), Wave attenuation by submerged vegetation: combining the effect of organism traits and tidal current, Mar. Ecol. Prog. Ser., 444, 31-41.

Pujol, D., T. Serra, J. Colomer, and X. Casamitjana (2013a), Flow structure in canopy models dominated by progressive waves, J. Hydrol., 486, 281-292.

Pujol, D., X. Casamitjana, T. Serra, and J. Colomer (2013b), Canopy-scale turbulence under oscillatory flow, Cont. Shelf Res., 66, 9-18.

Reidenbach, M., M. Limm, M. Hondzo, and M. Stacey (2010), Effects of bed roughness on boundary layer mixing and mass flux across the sediment-water interface, Water Resour. Res., 46(7).

Reidenbach, M. A., J. R. Koseff, and S. G. Monismith (2007), Laboratory experiments of fine-scale mixing and mass transport within a coral canopy, Phys. Fluids, 19(7), 075,107. 
Rominger, J. T., and H. M. Nepf (2014), Effects of blade flexural rigidity on drag force and mass transfer rates in model blades, Limnol. Oceanogr., 59(6), 2028-2041.

Ros, À., J. Colomer, T. Serra, D. Pujol, M. Soler, and X. Casamitjana (2014), Experimental observations on sediment resuspension within submerged model canopies under oscillatory flow, Cont. Shelf Res., 91, 220-231.

Rosman, J. H., J. R. Koseff, S. G. Monismith, and J. Grover (2007), A field investigation into the effects of a kelp forest (Macrocystis pyrifera) on coastal hydrodynamics and transport, J. Geophys. Res. Ocean., 112(C2).

Scott, L. C., J. W. Boland, K. S. Edyvane, and G. Jones (2000), Development of a seagrass-fish habitat model-I: A seagrass residency index for economically important species, Environmetrics, 11(5), 541-552.

Shimeta, J. (2009), Influence of flow speed on the functional response of a passive suspension feeder, the spionid polychaete Polydora cornuta, Mar. Biol., 156(12), 2451-2460.

Smith, N. M., and D. I. Walker (2002), Canopy structure and pollination biology of the seagrasses P. australis and P. sinuosa (Posidoneaceae), Aquat. Bot., 74(1), 57-70.

Stevens, C. L., C. L. Hurd, and P. E. Isachsen (2003), Modelling of diffusion boundary-layers in subtidal macroalgal canopies: The response to waves and currents, Aquat. Sci., 65(1), 81-91.

Thomas, C., and T. Foken (2007), Flux contribution of coherent structures and its implications for the exchange of energy and matter in a tall spruce canopy, Bound.-Lay. Meteorol., 123(2), 317-337.

Verduin, J. J., J. O. Backhaus, and D. I. Walker (2002), Estimates of pollen dispersal and capture within Amphibolis antarctica (Labill.) Sonder and Aschers. ex Aschers. meadows, Bull. Mar. Sci., 71(3), 1269-1277. 
Vogel, S. (1984), Drag and flexibility in sessile organisms, American Zoologist, 24(1), 37-44.

Wang, C., C. Wang, and Z. Wang (2010), Effects of submerged macrophytes on sediment suspension and NH sub (4)-N release under hydrodynamic conditions, J. Hydrodyn, 22(6), 810-815.

Zeller, R. B., F. J. Zarama, J. S. Weitzman, and J. R. Koseff (2015), A simple and practical model for combined wave-current canopy flows, J. Fluid Mech., 767, 842-880.

Zimmerman, R. C. (2003), A biooptical model of irradiance distribution and photosynthesis in seagrass canopies, Limnol. Oceanogr., 48(1, part2), 568-585.

Figure 1. The behavior of (a) model seagrass leaves (under the wave crest) with $B=7-50$, in comparison to a real $P$. australis leaf $(B \sim O(10)$ ). The model blade with $B=12$ exhibited the most realistic motion across all wave phases. In (b)-(d), fluid motion is from left to right (in the direction of wave propagation), while in (e)-(g), it is from right to left (in the offshore direction).

Figure 2. Vertical variation of frontal area in (a) real P. australis (collected from Shoalwater Bay, Western Australia) and (b) the model plants. (c) The lower region of the canopy has a greater frontal area than the upper region so, for model plants, the average of the upper and lower values of $a b\left(a b_{e f f}\right)$ was used to describe the canopy density.

Figure 3. Photographs showing the dye injection system in typical (a) rigid ( $a d=0.063)$ and (b) flexible $\left(a b_{\text {eff }}=0.064\right)$ canopies. Five fluorometers were arranged vertically to monitor the evolution of concentration profiles over depth.

Figure 4. The applicability of equations (5) and (6) in predicting the height of the center of the canopy shear layer $\left(l_{s l}\right)$ for flexible canopies in wave-dominated flows for both (a) $L<1$ and (b) $L>1$. The shaded area in the background represents the $95 \%$ confidence interval for the fitted curve. 
Figure 5. Vertical profiles of oscillatory velocity $\left(U^{r m s}\right)$ for an identical wave $\left(U_{\infty}^{r m s}=17 \mathrm{~cm} / \mathrm{s}\right.$ and $T=8 \mathrm{~s}$ ) over a medium (gray markers) and a high density canopy (black markers) for both (a) rigid and (b) flexible canopies. While an increasing velocity attenuation with canopy density is observed in both systems, flexible canopies generate a weaker velocity attenuation compared to the rigid analogues. Values of in-canopy ( $\left.U_{c}^{r m s}\right)$ and above-canopy RMS velocities $\left(U_{\infty}^{r m s}\right)$ and $\Delta U$ are indicated for the dense canopies. Gray dashed lines indicate the invariant height of rigid canopies and the effective height in the dense flexible canopy. Gray bands indicate the location of the shear layers in both canopies.

Figure 6. Comparison of the oscillatory velocity attenuation observed within rigid $\left(\Delta U_{R}\right)$ and flexible $\left(\Delta U_{F}\right)$ canopies subjected to identical waves. The dashed line indicates $1: 1$ agreement. There is a diminished attenuation in flexible canopies (relative to rigid canopies with equivalent dimensionless frontal areas) by, on average, $65 \%$.

Figure 7. Vertical profiles of normalized (a) $\overline{|u|^{\prime}}$ and (b) $T K E$ induced by an identical wave (Run 1, Table 3) over rigid and flexible canopies with $K C>5$. Black and gray dashed lines represent the center of the shear layer in rigid and flexible canopies, respectively. Reynolds stresses peak near the center of the shear layer in both environments, indicating enhanced vertical mixing of momentum in this region. Turbulent kinetic energy is greater within the canopy than above it due to the presence of wake turbulence. The particularly significant peak of $T K E$ within the shear layer in flexible canopies suggests the generation of strong turbulent fluctuations due to the plant motion.

Figure 8. Phase-averaged statistics for an identical wave over comparable dense rigid and flexible canopies (runs 1-RH and 1-FH), with $K C>5$ in both cases. (a) The in-canopy velocity leads the above-canopy velocity by a phase angle of up to $\pi / 6$ in rigid canopies. This phase lag is less pronounced in (b) flexible canopies due to the reduced drag in these environments. Enhanced momentum transfer is observed under the wave crest $(\varphi=0)$ in both (c) rigid and (d) flexible canopies. While a significantly enhanced turbulent kinetic energy is observed under the wave crest $(\varphi=0)$ and trough $(\varphi= \pm \pi)$ in (e) rigid canopies, $\langle T K E\rangle$ shows a more uniform distribution across all wave cycles in (f) flexible canopies.

Dashed lines represent the height of the center of the shear layer.

This article is protected by copyright. All rights reserved. 
Figure 9. Spectra of vertical turbulent velocity $\left(S_{w^{\prime} w^{\prime}}\right)$ in rigid and flexible canopies with $K C<5$ and $K C>5$. The estimated frequency bands for $\mathrm{KH}$-vortices and wake turbulence are indicated with light gray and dark gray bands, respectively. While the in-canopy spectrum demonstrates a negligible variation from those within the shear layer when (a) $K C<5$ (Run 13-RL), the magnitude of $S_{w^{\prime} w^{\prime}}$ is greatly enhanced within the shear layer for lower frequencies (gray arrow) when (c) $K C>5$ (Run 13-RH), supporting the generation of large scale shear-driven vortices. Similar results are observed in flexible canopies with (b) $K C<5$ and (d) $K C>5$ (runs 13-FL and 13-FH, respectively). The contribution of wake turbulence (black arrows) at higher frequencies is significant, for both rigid and flexible canopies.

Figure 10. (a) The applicability of eq. (16) for flexible canopies. There is a good agreement between measured values of $\left(D_{t, z}\right)_{F l e x i b l e}$ and the model proposed by Abdolahpour et al. (2017a) $\left(\left(D_{t, z}\right)_{\text {Predicted }}\right)$. (b) Vertical turbulent diffusivities are invariably lower within the flexible canopies relative to those observed within the corresponding rigid canopy $\left(\left(D_{t, z}\right)_{\text {Rigid }}\right)$ (on average 35\%). Gray and black markers represent canopies with medium and high density, respectively. Vertical and horizontal bars represent the standard deviation of diffusivity estimates from the $3-8$ replicate experiments undertaken in flexible and rigid canopies, respectively. In some cases, this variation is less than the marker size.

Table 1 Typical values for 100 P. australis leaves collected from two different sites (Cockburn Sound and Shoalwater Bay) in Western Australia.

\begin{tabular}{|l|l|l|}
\hline Variable & Average & Range \\
\hline$I(\mathrm{~cm})$ & $35 \pm 5$ & $27-43$ \\
\hline$t(\mathrm{~mm})$ & $0.3 \pm 0.06$ & $0.21-0.41$ \\
\hline$b(\mathrm{~cm})$ & $1.1 \pm 0.09$ & $0.92-1.1$ \\
\hline$m(\mathrm{~g})$ & $1.0 \pm 0.4$ & $0.6-1.7$ \\
\hline & & \\
\hline
\end{tabular}

Table 2 Canopy densities employed in this study.

This article is protected by copyright. All rights reserved. 


\begin{tabular}{|l|l|l|l|l|}
\hline Canopy type & Density & $a d, a b_{\text {eff }}$ & $L_{D}(\mathrm{~m})$ & $L_{c}(\mathrm{~m})$ \\
\hline & & & & \\
\hline & L (Low) & 0.016 & 0.39 & 9 \\
\hline Rigid (R) & M (Medium) & 0.063 & 0.10 & 5 \\
\hline & H (High) & 0.131 & 0.05 & 3 \\
\hline Flexible (F) & L (Low) & 0.015 & 0.71 & 9 \\
\hline & M (Medium) & 0.064 & 0.16 & 6 \\
\hline & H (High) & 0.145 & 0.07 & 6 \\
\hline & & & & \\
\hline
\end{tabular}

Table 3 Wave conditions to which the canopies were exposed.

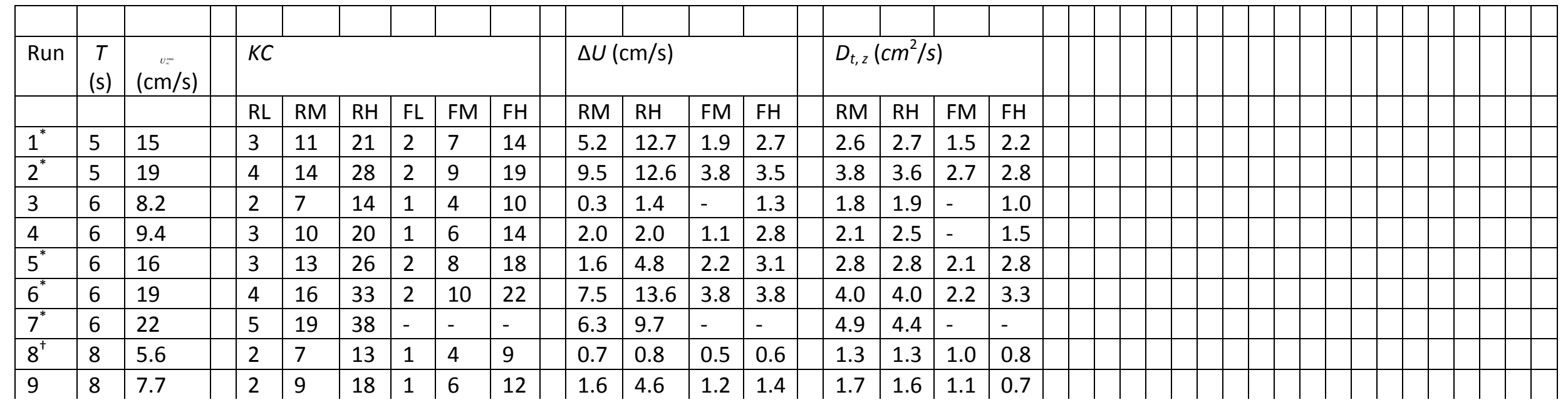

This article is protected by copyright. All rights reserved. 


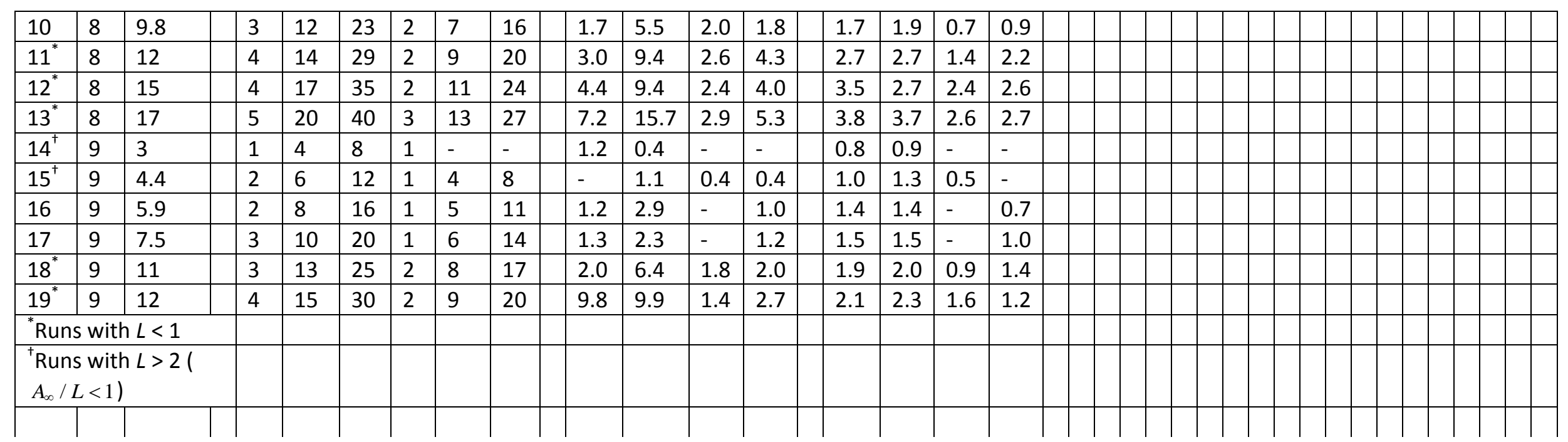

This article is protected by copyright. All rights reserved. 


\section{University Library}

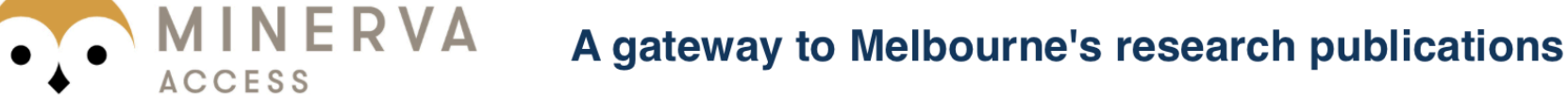

Minerva Access is the Institutional Repository of The University of Melbourne

Author/s:

Abdolahpour, M;Ghisalberti, M;McMahon, K;Lavery, PS

Title:

The impact of flexibility on flow, turbulence, and vertical mixing in coastal canopies

Date:

2018-11-01

Citation:

Abdolahpour, M., Ghisalberti, M., McMahon, K. \& Lavery, P. S. (2018). The impact of flexibility on flow, turbulence, and vertical mixing in coastal canopies. LIMNOLOGY AND OCEANOGRAPHY, 63 (6), pp.2777-2792. https://doi.org/10.1002//no.11008.

Persistent Link:

http://hdl.handle.net/11343/284750 